\title{
Compartir la mesa. Diseño centrado en personas para recursos museográficos educativos
}

Nayeli Zepeda Arias por mais de dez anos, tem colaborado com instituições públicas e privadas no México e nos Estados Unidos. Coordena o NodoCultura, um coletivo multidisciplinar que explora práticas comuns e dissidentes em museus. Faz parte do Museu Empathetic, uma iniciativa dedicada à construção de museus diversos, equitativos, acessíveis e inclusivos. Autora de textos sobre mediação, teoria e exercício museológico em publicações acadêmicas e independentes, tutora de programas de treinamento e de formação profissional e consultora em planejamento estratégico de áreas educacionais de museus e coleções. Historiadora de Arte (Universidade Ibero-Americana) e Mestre em Museografia Interativa e Didática (Universidade de Barcelona).<nayeli.zepeda@gmail.com> ORCID: 0000-0002-9117-6735
Resumen El diseño de recursos educativos para el museo es un proceso de mediación, y como tal, su mayor consideración son las personas. ¿Cómo podemos conectar con nuestros visitantes? ¿Cómo descifrar sus razones de significación? ¿Cómo integrar la participación y presencia de los usuarios en el desarrollo de esa mediación? El museo del siglo XXI se configura a partir de vínculos resilientes con sus visitantes y comunidad, y en la expansión de su ejercicio no considera únicamente su propia disciplina; por lo que podemos desarrollar acercamientos que, haciendo de la investigación, la empatía y el apoyo mutuo un hábito, concreten las intenciones comunicativas del museo y las necesidades de sus usuarios de forma significativa. En este texto se plantea el diseño centrado en personas como una estrategia para compartir la mesa con los visitantes, conocer lo que piensan, sienten y hacen, y establecer procesos colaborativos en la construcción del museo. En esta mesa, todos somos capaces de aprehender y aportar, por lo que se requiere de responsabilidad para reaprender y construir

Palabras clave Diseño centrado en personas, Design Thinking, Recursos educativos, Visitantes, Usuarios, Educación en museos. 


\section{Sharing the table. Human-centered design for museum education resources}

Abstract The design of museum education resources is a mediation process, and as such, its main considerations involves the visitors. How can we connect with our audiences? How to decode their reasons for meaning-making? How to integrate the users' participation and presence in the development of such mediation? The twenty-first-century museum is built on resilient links within its visitors and community. And in the expansion of its exercise, it does not only consider its discipline. By setting research, empathy, and mutual support, as a habit, we can develop approaches to reach the museum communications goal and the users' needs in a meaningful way. In this text, the human-centered design is meant as a strategy to share the table with the visitors, to know their thoughts, feelings, and doings, hence, to set up collaborative processes in the museum-making. At this table we are all able to apprehend and contribute, therefore, it takes responsibility to re-learn and build.

Keywords Human-centered design, Design Thinking, Educational resources, Visitors, Users, Museum Education.

\section{Compartilhe a mesa. Design centrado nas pessoas para recursos educacionais em museus}

Resumo 0 projeto de recursos educacionais para o museu é um processo de mediação e, como tal, sua maior consideração são as pessoas. Como podemos nos conectar com nossos visitantes? Como decifrar suas razões de importância? Como integrar a participação e presença de usuários no desenvolvimento dessa mediação? O museu do século XXI baseia-se em elos resilientes com seus visitantes e comunidade, e na expansão de seu exercício não considera apenas sua própria disciplina. Assim, podemos desenvolver abordagens que, tornando a pesquisa, a empatia e o apoio mútuo um hábito, especifiquem as intenções comunicativas do museu e as necessidades de seus usuários de maneira significativa. Neste texto, o design centrado nas pessoas é apresentado como uma estratégia para compartilhar a mesa com os visitantes, aprender o que pensam, sentem e fazem e estabelecer processos colaborativos na construção do museu. Nesta mesa, todos somos capazes de apreender e contribuir, por isso é necessário reaprender e construir.

Palavras Chave Design centrado nas pessoas, Design Thinking, Recursos educacionais, Visitantes, Usuários, Educação em museus. 


\section{Introducción}

La enunciación dogmática y enciclopédica del museo moderno aquél centrado en los objetos que expone y dicta el conocimiento- se ha abandonado por una narrativa múltiple y multiplicadora que reconoce a la institución museal como un espacio abierto en el que interviene una versatilidad de enunciaciones e interpretaciones. Este desplazamiento no es una aseveración binaria, sino que reconoce al museo como espacio patrimonial que significa y negocia.

Las estrategias como la curaduría educativa, la mediación y la integración de prácticas de otras disciplinas como el diseño centrado en personas, sugieren que más que ponderar la importancia entre las colecciones, el discurso académico o los públicos, se trata de un museo crítico, consciente, transparente, expandido, inclusivo y compartido. Es así como el museo actual tiene la encomienda de trazar una pluralidad de puntos de acceso y reconocer las subjetividades que fluyen como gestoras y generadoras de la misma institución (MONTERO, 2012).

Este texto ${ }^{1}$ aborda el diseño centrado en personas como un acercamiento que integra a los y las visitantes en los procesos museológicos, con el fin de entender y conectar con ellos, descifrar, negociar y articular sus razones de significación, y descubrir -en conjunto- oportunidades de comunicación y aprendizaje.

\section{Compartir la mesa}

La mesa es el espacio en el que se desarrollan actividades sociales, de comunidad, de cercanía, incluso de pertenencia: Compartimos la mesa entre amistades, con personas que desconocemos, juntas, en la presencia de otras y con otras, conversamos, jugamos, trabajamos, discutimos, planeamos, intercambiamos, establecemos límites. La socióloga Alice P. Julier señala que, al compartir la mesa con otros (as), dentro de un contexto cotidiano, las desigualdades sociales, económicas, entre otras, pierden importancia (Julier, 2013). No es que seamos iguales, sino que, ejerciendo los distintos niveles de intimidad, las diferencias no restringen las formas de interacción e intercambio, sino que las revelan.

En el postmuseo (HOOPER-GREENHILL, 2000) constructivista (HEIN, 2004), el visitante es activo en los procesos de comunicación e interpretación de las colecciones, aunque aún de manera moderada. El museo actual -y futuro-, expandiendo su propósito educativo, se concibe como un recurso esencial en sus comunidades (RUFFO, 2014), quienes lo emplean y del que disponen. Se modifica el entendimiento de la institución museal como la que dicta, ejecuta y posee el conocimiento; y cada agente (profesionales, visitantes o comunidades) establece una relación de confianza mutua y asume una responsabilidad conjunta o autoridad compartida (FRISCH, 2011; DUCLOS-ORSELLO, 2013; SALGADO, 2013). Este concepto niega la considera- 
ción de una única versión sobre el objeto de estudio, y apunta a un proceso de co-creación que se desarrolla a partir de la diversidad de fuentes, pero encaminadas a un propósito común: la aportación de valor al patrimonio y la experiencia. Es así como, en esta alianza, el museo y sus contenidos (textos, materiales, recursos) mutan con sus usuarios.

\section{Diseño centrado en personas: Hacia recursos compartidos}

La forma usual de desarrollo de materiales educativos y recursos de aprendizaje $^{2}$ se plantea desde una intencionalidad pedagógica que valora la participación -generalmente controlada-del público y la disposición los contenidos expositivos en distintos niveles ${ }^{3} \mathrm{o}$ capas de información y significación ${ }^{4}$; sin embargo, en el examen de lo que hacemos ¿cómo se reconoce la subjetividad y heterogeneidad de los y las visitantes? ¿cómo compartir la autoridad en el desarrollo de los recursos? ¿cómo responden nuestras prácticas al modelo de un museo consciente, inclusivo y compartido?

Desprenderse de la autoridad, compartir la mesa, crear relaciones democráticas, no es simplemente un acto de intervención consecuente de lo que el museo determina en los paneles, muros de opinión, cédulas didácticas, información ya publicada, ciertas exposiciones participativas (KOKE \& RYAN, 2017), sino considera la integración de los y las visitantes en la misma generación de herramientas de construcción de sentido y el modo de operar del museo. El diseño centrado en personas ${ }^{5}$ provee esta posibilidad no solamente en el desarrollo de recursos o materiales museográficos educativos, sino para escuchar, entender y aprender con nuestros públicos, al mismo tiempo que participan en las estructuras y narrativas del museo.

En Estados Unidos e Inglaterra, han empezado a utilizar formalmente este acercamiento para integrar a sus públicos a la construcción de sus propias experiencias. Y, en algunos museos de México, se realizan acercamientos que retoman el diseño centrado en personas y ensayos menores bajo responsabilidad personal de diseñadores y educadores.

Enfocado en el ser humano, este método es creativo, exploratorio, colaborativo, optimista y experimental; parte de las necesidades (prácticas, culturales, emocionales, intelectuales) de las personas para las que se diseña, las posibilidades con las que se cuenta y los requerimientos para que el producto, servicio o experiencia sea exitosa -significativa y relevante. Este proceso se fundamenta en la investigación y el conocimiento profundo de los usuarios y sus formas de interactuar, resolver, construir.

La iteración es una de sus condiciones: La práctica de prueba y error que empíricamente llevamos a cabo en el museo se formaliza, se mide y se hace habitual. El proceso de diseño varía según la escuela y organización ${ }^{6}$ que lo propone, e igualmente las técnicas que se implementan, pero las acciones base son consistentes e incluso agrupables: 
- Fase 1. Inspiración

Además de rescatar los procesos y soluciones que han desarrollado otras instituciones, este momento consiste en observar y estudiar a nuestros visitantes/usuarios, cómo se mueven en el espacio, qué recursos utilizan, qué herramientas necesitan, cómo conciben el museo, cómo son diferentes los unos de los otros en sus procesos de aprendizaje, qué piensan, qué esperan, qué buscan.

- Fase 2. Ideación

Así como la diversidad de nuestros visitantes, un problema no tiene una única solución, por lo que, este paso implica encontrar múltiples soluciones creativas, flexibles, posibles $\mathrm{y}$, aparentemente, imposibles; refinarlas y limitar ese grupo de ideas a la más factible, y trabajar sobre ella.

\section{- Fase 3. Implementación}

La idea se convierte en un prototipo, modelo experimental simple para probar o validar ideas, suposiciones, usabilidad, etc. Su conceptualización es rápida y austera para que se puedan hacer los cambios y mejoras de forma fácil.

- Fase 4. Evaluación

Bajo las mismas premisas de empatía y colaboración, la contribución y retroalimentación de los usuarios es crítica. Sin su perspectiva, no es posible escalar los resultados. Respondiendo a la condición cíclica de este acercamiento, la evaluación (diagnóstica, formativa, remedial y sumativa) sucede en todas las fases, y puede ser tan colaborativa e incluyente como sea posible.

Inspiración: Empatía como hábito

...el diseño es una actividad esencialmente social, actividad que no es posible separar ni abstraer del contexto de la vida de las personas... (KRIPPENDORFF, 2007, p. 4).

La empatía es un fin razonado más que un impulso vago (KONNIKOVA, 2012): No se concreta a un sentimiento personal (empatía emocional), sino que también involucra el entendimiento de lo que ve, dice, siente y piensa el otro, es el respeto por su experiencia, tiempo, esfuerzo, pensamientos y perspectivas (empatía cognitiva). Como consecuencia del sentir y pensar, existe un tercer componente, que consiste en actuar, trascendiendo la parte personal hacia un acto social (ZAKI \& OCHSNER, 2012). 
El diseño centrado en personas puede servirse de una gran diversidad de estrategias: observación y seguimiento (shadowing), desarrollo de personas ${ }^{7}$, entrevistas etnográficas, grupos focales, estudios cuantitativos, cualitativos, participativos. Es así como la empatía no es un elemento abstracto y aislado, sino que requiere de un punto de vista objetivo para determinar información concreta, útil y sustancial.

Considerada por el Center of the Future of Museums y la American Alliance of Museums como una de las tendencias de los museos en 2017 (AMERICAN ALLIANCE OF MUSEUMS, 2017), no es solamente una herramienta hacia fuera del museo, que posibilita un cambio en nuestros visitantes, sino que es posible construir una institución de forma empática. El primer paso para hacer de la empatía un hábito en el ejercicio museológico -sea cual sea nuestra posición- es aclarar nuestros propósitos, salir del escritorio, dejar de suponer, no confiar en primeras impresiones o elementos superficiales, analizar los detalles y aquello que pasamos por alto ${ }^{8}$, indagar qué es lo que realmente sucede en las salas, recorrer los espacios que recorren los y las visitantes, hablar con ellos (as), escucharles, conocerles y reconocerles, aprender sobre sus dificultades y diferencias, entender sus formas de interacción, descubrir qué es lo que les importa, ver desde su perspectiva, indagar sobre lo que dicen y lo que no dicen, sumergirse en sus experiencias.

\section{Ideación: El diseño es investigación}

Klaus Krippendorff sugiere que el diseño centrado en personas se define a partir de la concepción de futuros posibles, mutables, deseables y accesibles; los caminos reales y factibles para llegar a esas promesas; y el plan de acciones coordinadas para lograrlo (KRIPPENDORFF, 2007). La fase de ideación comprende precisamente esas posibilidades que tal vez no se habían considerado: Se trata de interpretar y explicar las perspectivas y narrativas que se encontraron anteriormente; dar sentido a los hallazgos (materiales muy especializados, lenguaje adecuado, buen funcionamiento, etcétera) y establecer las rutas para desarrollar el recurso, espacio, experiencia que es realmente necesario.

La investigación es la búsqueda de un conocimiento que extiende los límites de lo que ya se sabe; y más allá del objeto museológico de estudio, su fin es mejorar la práctica. El diseño centrado en personas es investigación, así también el desarrollo de recursos museográficos y educativos. Si consideramos que una exposición no inicia y concluye en su montaje, tampoco las herramientas que la construyen y soportan.

En 2016, el Museo de Arte de Phoenix (Estados Unidos) realizó un sprint $^{9}$ de un día para desarrollar una guía de visita impresa, con la intención de desmitificar la visita al museo. Sus primeras preguntas eran por qué la gente visita los museos, por qué se toman selfies y las comparten socialmente; la respuesta: para demostrar por qué están ahí. Así partieron desde la idea "estoy aquí"; y definieron tres contextos: por primera vez, en 
familia, en una cita. Al revisar los estudios con los que ya contaban (demográficos y evaluaciones), la bibliografía de John Falk en cuanto a las motivaciones de los y las visitantes, en pocas horas el equipo -de forma individual y con revisiones grupales- se aventuró a escribir breves recorridos. Realizaron 200 copias (como parte del primer prototipo -pues actualmente en dos meses reparten alrededor de 300) de las guías I'm here para poner a evaluación pública. Después de ese primer lanzamiento, las modificaciones han sido constantes: son más extensas, incluyen imágenes de más objetos y son bilingües; además, se ha creado una nueva guía para maestros. En el interés de tener más información, se han registrado quiénes y qué guías usan, los días y horas en las que más se solicitan, cantidad de guías usadas, entre otros aspectos ${ }^{10}$.

\section{Implementación: De la idea al prototipo}

La elaboración y uso de prototipos (bocetos, esquemas, maquetas, modelos a escala, señalética temporal, dummies) es una propuesta de desarrollo museográfico que se inició en los museos de ciencia, particularmente en el Exploratorium de San Francisco (1970s) y, en México, Papalote Museo del Niño se fundó básicamente como uno (1992).

En el Museo Interactivo de Economía (México), dentro de la Dirección de Desarrollo de Experiencias y la Jefatura de Diseño, realizan prototipos de diversos tipos con el propósito de probar funcionalidad, usabilidad, contenido, mensaje y formato de sus interactivos. Con el apoyo de la Subdirección de Estudios de Público y Evaluación, estas muestras se examinan con público real en un espacio de prueba y estabilización ${ }^{11}$. De manera similar, desde 2013, el Museo de Arte de Indianápolis (Estados Unidos) ha trabajado el diseño de exposiciones centradas en el público y, cuenta con un laboratorio donde sus usuarios pueden experimentar con prototipos de aplicaciones digitales. A partir del mismo año, el Museo de Arte de Milwaukee (Estados Unidos) realizó un ejercicio para que sus públicos modelaran su experiencia en el museo.

Kathleen McLean, una de las profesionales/consultora de museos más respetada en el mundo, ha escrito generosamente sobre el uso de prototipos en el museo como una forma de trabajo que se enraíza en la estructura organizacional. Concibe los museos como incubadoras controladas en las que se experimenta con exposiciones y elementos museográficos (cédulas, materiales educativos, espacios y dispositivos interactivos, espacios lúdicos) donde el propósito es probar el proceso y la relevancia de las ideas y objetos. McLean explica que se empieza con una idea pequeña (una sala, un objeto, un tema) que ha de resolverse de forma austera, rápida y en grupos pequeños, pero involucrando a expertos (as) y visitantes para conversar y reflexionar en conjunto (MCLEAN, 2015, 2017). 


\section{Evaluación: Aprender juntos (as)}

La evaluación hace posible que el aprendizaje sea constante, dinámico, modificable; y su deriva es el cambio, sino no tiene sentido. No solamente importa para la experiencia en la que se está trabajando (si es útil, funcional, relevante, accesible), sino que permite compartir lo que hacemos con profesionales que están realizando las mismas búsquedas, y con nuestra comunidad. Transparentar el proceso, llevarlo a la arena pública, también enriquece el museo a través de la suma de participantes, colectivos y voces profesionales, del apoyo de mecenas y patrocinadores, y la acreditación de directivos y otras organizaciones.

Evaluar los recursos museográficos educativos requiere de observación, de escucha e, invariablemente, de empatía y, por tanto, de acción. Una vez construidos los prototipos museográficos, la retroalimentación de las personas que interactuarán con ellos implica reexaminar qué ven, sienten, hacen, dicen, y también lo qué no dicen de su experiencia, sus gestos y silencios (SCHMILCHUCK, 2017).

El y la profesional de museo realizan múltiples tareas, particularmente, los equipos educativos -a veces de una o dos personas- investigan, diseñan, median, en tiempos y presupuestos límites; lo que implica que muchas veces, trabajan a partir de primeros o superficiales acercamientos, improvisando formas de evaluación y suponiendo sus resultados. Sin embargo, podemos contar con los y las visitantes como aliados (as) comprometidos (as) con esa misma labor.

Como parte del rediseño de la galería dedicada a California del Museo de Oakland, se evaluaron los textos, aprovechando que son elementos que se pueden modificar fácil y rápidamente. Encontraron una forma muy fructífera de llevar a cabo la fase de evaluación en conjunto con sus visitantes: Convocar un pequeño grupo interdisciplinario de profesionales vinculados al museo y de visitantes asiduos para captar retroalimentación, comentarios, e información (OAKLAND MUSEUM OF CALIFORNIA, 2010). Se emplearon prototipos, probaron con entrevistas, grupos focales y encuestas; se implementó un programa específico para la revisión de estos recursos y una guía de lineamientos para su desarrollo. 


\section{¿Es posible que la transformación deje de ser un lugar común para que sea acción?}

La realidad de nuestros museos es que a múltiples niveles se requiere de negociaciones, transparencia y evidencia para mediar y resarcir los intersticios que deja el complejo sistema museístico; pero ello no puede articularse sin una red que sostenga y promueva la investigación, la empatía, la experimentación y la colaboración.

El diseño centrado en personas es un instrumento que permite que, en el proceso dialógico, iterativo y social de generación de herramientas de construcción de sentido (de recursos museográficos y/o educativos, programación), el modo de operar del museo y la estructura institucional se configure a partir de la asociación emocional, cognitiva y activa entre la institución y su comunidad.

Las personas son el punto de partida: encontrarlas, escucharlas, intercambiar con ellas, desplegar la diversidad de nuestras comunidades y sus narrativas, celebrar sus diferencias, reconocer sus aportaciones y contribuciones, es la ruta hacia la transformación. Si buscamos que los cambios sean duraderos, en los que la institución museal sea realmente relevante y social, entonces tenemos que compartir la autoridad y ceder al visitante un espacio -y más- en la mesa, en la que todos (as), responsables, juntos (as) en la revelación de nuestra diversidad, seamos capaces de aprehender, aportar, reaprender, construir y reconstruir(nos).

\footnotetext{
1 Este texto fue originalmente presentado como la conferencia "Compartir la mesa. Diseño centrado en personas para recursos museográfcos educativos", en el marco del "Simposio Internacional: Ocio, Museos e Igualdad", en el Centro Cultural España, Ciudad de México, octubre 2017. 2 Considerando los recursos educativos como dispositivos comunicacionales utilizados con intencionalidad pedagógica; mientras los materiales didácticos se diseñan y emplean desde el inicio para el desarrollo de acciones didácticas, y se dirigen especialmente a los docentes, maestros (as) o aquéllos (as) profesionales que llevan a cabo los procesos de enseñanza. En ambos casos, para los museos, no son simplemente una pauta de información al público (experto o no), sino una herramienta que promueve, media y mejora la experiencia de visitar, usar y habitar el museo; son medios que tienen el propósito de facilitar una mayor comprensión de su contenido expositivo, de sus colecciones y enunciaciones; e incluyen desde elementos escritos como hojas de sala, textos en muro, guías de visita, hasta programas, audiovisuales y ambientes, como espacios lúdicos y estrategias experimentales y participativas.
} 
3 No confundir con organización de contenidos.

4 Fundación TyPA cuenta con una guía práctica que aborda la dinamización de exposiciones ya montadas y el diseño de estrategias, acciones y materiales que integran a los y las visitantes en su creación y desarrollo. Esta propuesta resultó del Laboratorio TyPA de Gestión en Museos 2013, en conjunto con MUS, red de profesionales de museos, y con la participación de Tam Muro, Elaine Heumann Gurian, Jim Volkert, entre otros expertos.

5 El diseño centrado en personas está altamente relacionado con el diseño empático, y el diseño participativo o co-diseño.

6 Los procesos y términos también varían según si refiere estrictamente a design thinking (d.school de Stanford), diseño centrado en personas (IDEO), diseño centrado en usuarios (más utilizado al ámbito tecnológico).

7 Las "personas" son elementos ficticios que representan perfiles de diferentes usuarios, y se crean con base en la investigación que se ha realizado sobre cómo interactúan, resuelven o usan un producto o servicio. 8 En 1991, el estudio de diseño Doblin (Estados Unidos) desarrolló el modelo AEIOU para interpretar las observaciones recopiladas en los procesos de diseño centrado en personas: A (actividades), E (entorno), I (interacciones), 0 (objetos) y U (usuarios).

9 Es un proceso similar para responder problemas críticos a través de la investigación, prototipar, y probar ideas con los y las visitantes; pero éste se hace de manera aún más rápida, en cuestión de horas o días.

10 Información proporcionada por Christian Adame, director asistente de educación del Museo de Arte de Phoenix (correo personal, septiembre de 2017).

11 Información proporcionada por Alberto Rojas, jefe de diseño del Museo Interactivo de Economía (correo personal, septiembre de 2017).

\section{Referencias}

AMERICAN ALLIANCE OF MUSEUMS (coord.). Trendswatch 2017. In: Alliance Labs, 2017. Disponível em: http://labs.aam-us.org/trendswatch2017/. Acesso em: 10 de janeiro de 2017. DUCLOS-ORSELLO, E. Shared Authority: The Key to Museum Education as Social Change. In: Journal of Museum Education, v. 38, n. 2, p. 121-128, 2013.

FRISCH, M. From A Shared Authority to the Digital Kitchen, and Back. In: Letting Go? Sharing Historical Authority in a User-Generated World. Philadelphia: The Pew Center for Arts and Heritage, 2011, p. 126-137.

JULIER, A. Eating Together. Food, Friendship, and Inequality. Chicago: University of Illinois Press, 2013.

KOKE, J.; RYAN, K. From Consultation to Collaboration. Mechanisms for Integrating Community Voices into Exhibition Development. In: Visitor-Centered Exhibitions and Curation in Art Museums. Londres: Rowman \& Littlefield, 2017, p. 47-56.

KONNIKOVA, M. The empathy machine. In: AEON Magazine, 2012. Disponível em: https:// aeon.co/essays/empathy-depends-on-a-cool-head-as-much-as-a-warm-heart.

KRIPPENDORFF, K. Design research, an oxymoron? In: R. Design research now. Essays and selected projects. Basel: Birkhäuser, 2007, p. 67-80.

MCLEAN, K. Learning to Be Nimble: Museum Incubators for Exhibition Practice. In: Exhibitionist, v. 13, p. 8-13, 2015. 
MCLEAN, K. Prototipos en museos: creación de exposiciones más interesantes con un proceso de evaluación distinto. In: Estudios sobre públicos y museos. Volumen II. Apuntes para pasar de la teoría a la práctica. Ciudad de México: INAH/ENCRyM, 2017, p. 96-106.

OAKLAND MUSEUM OF CALIFRONIA. How visitors changed our museum, 2010. Disponível em: http://museumca.org/files/HowVisitorsChangedOurMuseumBook.pdf. Acesso em: 10 de janeiro de 2017.

RODRIGO, J. Experiencias de mediación crítica y trabajo en red en museos: de las políticas de acceso a las políticas en red. In: Academia, 2012. Disponível em: https://www.academia. edu/3537028/Experiencias_de_mediación_crítica_y_trabajo_en_red_en_museos_de_las_ políticas_de_acceso_a_las_políticas_en_red. Acesso em: 10 de janeiro de 2017.

RUFFO, C. Museums 3.0: Implementing programs/exhibits which are a community resource. En: Association of Science - Technology Centers, 2014. Disponível em: http://www. astc.org/annual-conference/museums-3-0-implementing-programsexhibits-which-are-a-community-resource/. Acesso em: 10 de janeiro de 2017.

SALGADO, M. Diseñando un museo abierto. In: Wolkowicz Editores, 2013. Disponível em: http://www.wolkowiczeditores.com.ar/libros_digitales/museo_abierto_salgado.pdf. Acesso em: 10 de janeiro de 2017.

SAMIS, P.; MICHAELSON, M. Creating the Visitor-Centered Museum. Nueva York: Routledge., 2016

SCHMILCHUCK, G. Dos caras de una moneda. Evaluar exposiciones y museos/conocer los públicos. In: Estudios sobre públicos y museos. Volumen II. Apuntes para pasar de la teoría a la práctica. Ciudad de México: INAH/ENCRyM, 2017, p. 40-49.

ZAKI, J.; OCHSNER, K. N. The neuroscience of empathy: progress, pitfalls and promise. In: Nature Neuroscience, v. 15, n. 5, p. 675.680, 2012. 